\title{
Distributed and Decentralized Kalman Filtering for Cascaded Fractional Order Systems
}

\author{
Martin Kupper ${ }^{1,2}$, Iñigo Sesar Gil ${ }^{1}$ and Sören Hohmann ${ }^{1}$
}

\begin{abstract}
This paper presents a distributed Kalman filter algorithm for cascaded systems of fractional order. Certain conditions are introduced under which a division of a fractional system into cascaded subsystems is possible. A functional distribution of a large scale system and of the state estimation algorithm leads to smaller and scalable nodes with reduced memory and computational effort. Since each subsystem performs its calculations locally, a central processing node is not needed. All data which are required by subsequent nodes are communicated to them unidirectionally. Also a comparison between the Fractional Kalman Filter (FKF) and the Cascaded Fractional Kalman Filter (CFKF) is given by an example.
\end{abstract}

\section{INTRODUCTION}

\section{A. Fractional Systems}

During the last decades, fractional calculus has gained considerable attention. Some characteristics of fractional calculus are the capability to predict the dynamical behavior of physical systems more precisely and the possibility to describe systems with simple models using only a few physically motivated parameters [1], [2].

Applications of fractional calculus are approaches for the identification of Li-ion battery parameters which avoid the usage of integer order approximations with more parameters. An example of a fractional order battery model is described in [2], which is introduced using impedance measurements. A late-lumping parameter identification method for a fractional battery model is presented in [3]. In [4] a model-based approach is derived which extends Mikusińskis operational calculus for fractional systems in order to identify the distribution of relaxation times of a battery cell. In [5] a spectral parameter estimation method is presented for the identification of a linear fractional state space system.

In control theory a fractional state space model [6] serves as a basis for a state-feedback control. Similar to integer order models, it is possible that not all states of a fractional system are measurable. Since the state estimation problem in fractional systems is more complicated than in integer order systems, a suitable estimation method for fractional order systems is required. For example, a fractional Kalman filter (FKF) is developed in [7] and an adaptive $H_{\infty}$ observer with a joint parameter identification method is presented in [8].

\footnotetext{
*This work was supported by the state of Baden-Württemberg and the ITK Engineering AG

${ }^{1}$ Martin Kupper, Iñigo Sesar Gil and Sören Hohmann are with the Institute of Control Systems, Karlsruhe Institute of Technology, Karlsruhe, Germany martin.kupper@kit.edu

${ }^{2}$ Martin Kupper is also with the ITK Engineering AG, Rülzheim, Germany
}

\section{B. Distributed and Cascaded Systems}

In many applications, all calculations are performed by a single processor. Therefore, all measurements and signals have to be transmitted to this processor. Additionally, the complete state vector has to be estimated in that processor, which is computationally intensive. These properties can cause errors in practical applications [9]. To avoid these problems, a functional decentralization and distribution procedure can be applied where the system is divided into smaller subsystems which communicate with each other. It achieves modularity, reduces complexity and lowers the computational cost in the particular subsystems.

The decentralization and distribution of the Kalman filter has been presented, for example in [9]-[11] for integer order systems and in [12] for fractional order systems. However, the distribution scheme can be complicated and laborious for some system classes, because the choice of appropriate transformation matrices and the implementation of an additional fusion step can be ambiguous and complex. A decomposition of the system into cascaded subsystems is more convenient sometimes, since the cascaded approach does not require transformation matrices and a fusion step. In [13] a method for a distributed Kalman filter for cascaded systems of integer order has been presented.

The main contribution of this paper is the development and the detailed deduction of a cascaded Kalman filter for systems of fractional order (CFKF). Further subjects are the extension of the approach to more than 2 subsystems and an improvement of the algorithm compared to [13]. An example compares the performance of the FKF with the CFKF. Additionally, the effect of a wrong initialization of the states is considered. In the future, we plan to apply this procedure to large battery packs where the particular cells are modelled using fractional systems. Further applications could be brain studies [14] where many sensors are used to observe the fractional-order dynamics of the brain.

\section{FRACTIONAL CALCULUS}

First, the Grünwald-Letnikov definition as a discrete-time representation of a fractional derivative [15] is introduced by

$$
{ }_{0} D_{t_{k}}^{q} x\left(t_{k}\right)=\Delta^{q} x_{k}=\frac{1}{h^{q}} \sum_{j=0}^{k}(-1)^{j}\left(\begin{array}{l}
q \\
j
\end{array}\right) x_{k-j}
$$

where $q \in \mathbb{R}^{+}$is the order of the fractional derivative, $k$ is the current sample of the sequence and $h$ is the sampling interval. The symbol $D$ is used to represent the fractional derivative of a function with the lower bound 0 and the upper 
bound $t_{k}$. This section deals with difference equations that use discrete sequences, and not with differential equations based on continuous functions. Therefore, according to the formula of the $q$-th order difference in (1), and also for simplicity reasons the value of $h$ is set to $h=1$ similar to [7]. Additionally, the term $x_{k}$ in (1) is isolated, resulting in

$$
x_{k}=\Delta^{q} x_{k}-\sum_{j=1}^{k}(-1)^{j}\left(\begin{array}{l}
q \\
j
\end{array}\right) x_{k-j} .
$$

Based on (2), the linear stochastic discrete fractional order state space system [7] can be obtained, which is defined by

$$
\begin{aligned}
\Delta^{\gamma} \boldsymbol{x}_{k} & =\boldsymbol{A} \boldsymbol{x}_{k-1}+\boldsymbol{B} \boldsymbol{u}_{k-1}+\boldsymbol{\omega}_{k-1} \\
\boldsymbol{x}_{k} & =\Delta^{\gamma} \boldsymbol{x}_{k}-\sum_{j=1}^{k}(-1)^{j} \boldsymbol{\Gamma}_{j} \boldsymbol{x}_{k-j} \\
\boldsymbol{y}_{k} & =\boldsymbol{C} \boldsymbol{x}_{k}+\boldsymbol{\nu}_{k} \\
\boldsymbol{\Gamma}_{j} & =\operatorname{diag}\left[\left(\begin{array}{c}
q_{1} \\
j
\end{array}\right), \cdots,\left(\begin{array}{c}
q_{N} \\
j
\end{array}\right)\right] \\
\Delta^{\gamma} \boldsymbol{x}_{k} & =\left[\begin{array}{c}
\Delta^{q_{1}} x_{1, k} \\
\vdots \\
\Delta^{q_{N}} x_{N, k}
\end{array}\right]
\end{aligned}
$$

where $\boldsymbol{x}_{k} \in \mathbb{R}^{N}$ is the state vector, $\boldsymbol{u}_{k} \in \mathbb{R}^{L}$ is the system input, $\boldsymbol{y}_{k} \in \mathbb{R}^{M}$ is the system output, $\boldsymbol{\omega}_{k} \in \mathbb{R}^{N}$ is the system noise, $\boldsymbol{\nu}_{k} \in \mathbb{R}^{M}$ is the output noise and $q_{1}, \ldots, q_{N} \in \mathbb{R}^{+}$are the orders of the particular system equations. A derivation of these equations from a continuoustime representation, where also the sampling interval $h$ is considered, is given in [6]. Of course, there are alternative descriptions for fractional systems, but these can directly be transferred into the equations from (3) to (7).

We assume that the system is bounded-input boundedoutput stable and observable [6]. Therefore, we make use of the observability condition from [16] which is defined as follows. The system modelled by equations (3) to (7) is observable in a finite time $K$, if the matrix

$$
\mathrm{O}_{K}=\left(\begin{array}{c}
C G_{0} \\
\vdots \\
C G_{K-1}
\end{array}\right)
$$

with

$$
\boldsymbol{G}_{k}= \begin{cases}\boldsymbol{I}_{N}, & k=0 \\ \sum_{j=0}^{k-1} \boldsymbol{A}_{j} \boldsymbol{G}_{k-1-j}, & k \geq 1\end{cases}
$$

has full rank. Note that contrary to integer order systems the $\operatorname{rank}\left(\mathbf{O}_{K}\right)$ can increase for $K \geq N$. Further information can be found in [16].

For the exact calculation of the fractional derivative, the initial values of the states have to be given from $t=-\infty$ to the current time $t=t_{k}=k h$ [17], [18]. So, actually the sums in equations (1), (2) and (4) must include the values from $j=1$ to $j=\infty$. But since in the practical implementation of such a filter the past values are usually unknown, we can deal only with the data given from $t=0$ on. A possible solution proposed by [18] to handle unknown initial values is to use $\Phi(t)$ with

$$
|\Phi(t)|<K t^{q-1}, \quad K \in \mathbb{R}^{+}
$$

as an initialization function when the system history is unknown. Alternatively, one may neglect the initialization function when the estimation algorithm starts while the system is in a position of rest for a period of time, since the free response decays towards 0 as $t^{q-1}$.

We also make use of the short memory principle, which means that we neglect values of the states that lie further in the past. If $f(t) \leq M$, then the error of that function caused by the short memory principle is bounded [6] by $e(t)$

$$
e(t) \leq \frac{M B_{L}^{-q}}{|\Gamma(1-q)|}
$$

with $B_{L}$ being the buffer or memory length. Therefore, a sufficient buffer length for a given error bound can always be found. For the following sections, it can be assumed that the buffer length is chosen sufficiently large. It should be noted that, according to [18], the fractional system described by (3) to (7) does not form a classical state space representation. It is rather a pseudo state space description, because it depends not only on the current state $\boldsymbol{x}_{k}$, but also on the values of $\boldsymbol{x}$ from the past as discussed in this section. However, for simplicity reasons, in the following sections the system described above is named "state space system".

\section{FRACTIONAL KALMAN FILTER}

The FKF for a linear stochastic discrete-time fractional order state space system [7] is given by

$$
\begin{aligned}
\Delta^{\gamma} \hat{\boldsymbol{x}}_{k}^{-} & =\boldsymbol{A} \hat{\boldsymbol{x}}_{k-1}^{+}+\boldsymbol{B} \boldsymbol{u}_{k-1} \\
\hat{\boldsymbol{x}}_{k}^{-} & =\Delta^{\gamma} \hat{\boldsymbol{x}}_{k}^{-}-\sum_{j=1}^{z}(-1)^{j} \boldsymbol{\Gamma}_{j} \hat{\boldsymbol{x}}_{k-j}^{+} \\
\boldsymbol{P}_{k}^{-} & =\left(\boldsymbol{A}+\boldsymbol{\Gamma}_{1}\right) \boldsymbol{P}_{k-1}^{+}\left(\boldsymbol{A}+\boldsymbol{\Gamma}_{1}\right)^{T} \\
& +\boldsymbol{Q}_{k-1}+\sum_{j=2}^{z} \boldsymbol{\Gamma}_{j} \boldsymbol{P}_{k-j}^{+} \boldsymbol{\Gamma}_{j}^{T} \\
\boldsymbol{K}_{k} & =\boldsymbol{P}_{k}^{-} \boldsymbol{C}_{k}^{T}\left(\boldsymbol{C}_{k} \boldsymbol{P}_{k}^{-} \boldsymbol{C}_{k}^{T}+\boldsymbol{R}_{k}\right)^{-1} \\
\hat{\boldsymbol{x}}_{k}^{+} & =\hat{\boldsymbol{x}}_{k}^{-}+\boldsymbol{K}_{k}\left(\boldsymbol{y}_{k}-\boldsymbol{C}_{k}^{-}\right) \\
\boldsymbol{P}_{k}^{+} & =\left(\boldsymbol{I}-\boldsymbol{K}_{k} \boldsymbol{C}\right) \boldsymbol{P}_{k}^{-}\left(\boldsymbol{I}-\boldsymbol{K}_{k} \boldsymbol{C}\right)^{T}+\boldsymbol{K}_{k} \boldsymbol{R}_{k} \boldsymbol{K}_{k}^{T} \\
& =\left(\boldsymbol{I}-\boldsymbol{K}_{k} \boldsymbol{C}\right) \boldsymbol{P}_{k}^{-}
\end{aligned}
$$

where $z=\min \left[k, B_{L}\right]$ stands for the upper limit of the sum. The FKF is usually initialized with a priori known values or

$\hat{\boldsymbol{x}}_{0}^{+}=E\left\{\boldsymbol{x}_{0}\right\} \approx \mathbf{0}$,

$\boldsymbol{P}_{0}^{+}=E\left\{\left(\boldsymbol{x}_{0}-\hat{\boldsymbol{x}}_{0}^{+}\right)\left(\boldsymbol{x}_{0}-\hat{\boldsymbol{x}}_{0}^{+}\right)^{T}\right\} \approx 100 \boldsymbol{I}$

when the initial values are unknown [7]. Alternatively one can use the initialization in (10) from [18]. $(*)^{-}$describes the a priori estimation and $(*)^{+}$the a posteriori estimation, $\boldsymbol{P}, \boldsymbol{R}, \boldsymbol{Q}$ are the covariance matrices of the estimated values, the measurement noise and the system noise, respectively. It is assumed that $\boldsymbol{P}, \boldsymbol{R}$ and $\boldsymbol{Q}$ are symmetric, $\boldsymbol{\nu}_{k}$ and $\boldsymbol{\omega}_{k}$ 
are uncorrelated and with zero expected value and $E\left\{\left(\hat{\boldsymbol{x}}_{\boldsymbol{j}}-\right.\right.$ $\left.\left.\boldsymbol{x}_{\boldsymbol{j}}\right)^{T}\left(\hat{\boldsymbol{x}}_{\boldsymbol{k}}-\boldsymbol{x}_{\boldsymbol{k}}\right)\right\} \approx 0$ for $j \neq k$. It can be seen in the equations that past estimates $\hat{\boldsymbol{x}}_{j}$ will not be updated when new data $\boldsymbol{u}_{k}$ or $\boldsymbol{y}_{k}$ with $j<k$ are obtained. Therefore, the FKF shown in (12) to (19) is a suboptimal state estimation algorithm. An algorithm which also updates and estimates past states is presented in [19]. This procedure can also be used for the CFKF as it is straightforward to adapt and to implement. More Details and the proof of the FKF can be found in [7].

\section{DISTRIBUTED STATE ESTIMATION OF CASCADED FRACTIONAL SYSTEMS}

In this section we want to introduce the Cascaded Fractional Kalman Filter (CFKF) for the distributed state estimation of cascaded fractional systems. A distributed and decentralized estimation scheme for fractional order systems is presented in [12], where the dependent states of different subsystems are merged in a fusion step. Advantages of this approach are reduced computational cost, improved efficiency, reliability and easier tuning for large scale systems.

However, the complete distribution procedure and the process of choosing appropriate transformation matrices can be cumbersome if the system is already in a cascaded form or when it is possible to transform it into cascaded subsystems. In this case the development of local cascaded estimators is more suitable. Consider [12] for further information about the distributed and decentralized fractional Kalman filter.

The main idea of the CFKF is that the state vectors in the subsystems contain only local states. All other states of the remaining subsystems that have a significant influence over their dynamics are taken into account in a separate term of the state equation in the estimation algorithm. Therefore, the CFKF is also a decentralized and distributed filter.

\section{A. Cascaded Subsystems}

It is required that $(\boldsymbol{A}, \boldsymbol{C})$ of system (3) to (5) is an observable pair using the criterion of equation (8) and can be described as block lower triangular matrices as follows

$$
\begin{aligned}
\left(\begin{array}{c}
\Delta^{\gamma_{1}} \boldsymbol{x}_{1, k} \\
\Delta^{\gamma_{2}}
\end{array} \boldsymbol{x}_{2, k}\right. & =\left(\begin{array}{cc}
\boldsymbol{A}_{11} & \mathbf{0} \\
\boldsymbol{A}_{21} & \boldsymbol{A}_{22}
\end{array}\right)\left(\begin{array}{l}
\boldsymbol{x}_{1, k-1} \\
\boldsymbol{x}_{2, k-1}
\end{array}\right)+ \\
& +\left(\begin{array}{l}
\boldsymbol{B}_{1} \\
\boldsymbol{B}_{2}
\end{array}\right) \boldsymbol{u}_{k-1}+\left(\begin{array}{l}
\boldsymbol{\omega}_{1, k-1} \\
\boldsymbol{\omega}_{2, k-1}
\end{array}\right) \\
\left(\begin{array}{l}
\boldsymbol{x}_{1, k} \\
\boldsymbol{x}_{2, k}
\end{array}\right) & =\left(\begin{array}{c}
\Delta^{\gamma_{1}} \boldsymbol{x}_{1, k}-\sum_{j=1}^{k}(-1)^{j} \boldsymbol{\Gamma}_{1, j} \boldsymbol{x}_{1, k-j} \\
\Delta^{\gamma_{2}} \boldsymbol{x}_{2, k}-\sum_{j=1}^{k}(-1)^{j} \boldsymbol{\Gamma}_{2, j} \boldsymbol{x}_{2, k-j}
\end{array}\right) \\
\left(\begin{array}{l}
\boldsymbol{y}_{1, k} \\
\boldsymbol{y}_{2, k}
\end{array}\right) & =\left(\begin{array}{ll}
\boldsymbol{C}_{11} & \mathbf{0} \\
\boldsymbol{C}_{21} & \boldsymbol{C}_{22}
\end{array}\right)\left(\begin{array}{l}
\boldsymbol{x}_{1, k} \\
\boldsymbol{x}_{2, k}
\end{array}\right)+\left(\begin{array}{l}
\boldsymbol{\nu}_{1, k} \\
\boldsymbol{\nu}_{2, k}
\end{array}\right)
\end{aligned}
$$

with the division of

$$
\boldsymbol{x}_{k}=\left(\begin{array}{l}
\boldsymbol{x}_{1, k} \\
\boldsymbol{x}_{2, k}
\end{array}\right), \boldsymbol{y}_{k}=\left(\begin{array}{l}
\boldsymbol{y}_{1, k} \\
\boldsymbol{y}_{2, k}
\end{array}\right), \boldsymbol{\Gamma}_{j}=\operatorname{diag}\left(\boldsymbol{\Gamma}_{1, j}, \boldsymbol{\Gamma}_{2, j}\right)
$$

into two subsystems for convenience and without loss of generality, similar to [13]. Then, the system is divided into

$$
\begin{aligned}
\Delta^{\gamma_{1}} \boldsymbol{x}_{1, k} & =\boldsymbol{A}_{11} \boldsymbol{x}_{1, k-1}+\boldsymbol{B}_{1} \boldsymbol{u}_{k-1}+\boldsymbol{\omega}_{1, k-1} \\
\boldsymbol{x}_{1, k} & =\Delta^{\gamma_{1}} \boldsymbol{x}_{1, k}-\sum_{j=1}^{z}(-1)^{j} \boldsymbol{\Gamma}_{1, j} \boldsymbol{x}_{1, k-j} \\
\boldsymbol{y}_{1, k} & =\boldsymbol{C}_{11} \boldsymbol{x}_{1, k}+\boldsymbol{\nu}_{1, k}
\end{aligned}
$$

and

$$
\begin{aligned}
& \boldsymbol{x}_{2, k}=\Delta^{\gamma_{2}} \boldsymbol{x}_{2, k}-\sum_{j=1}^{z}(-1)^{j} \boldsymbol{\Gamma}_{2, j} \boldsymbol{x}_{2, k-j} \\
& \boldsymbol{y}_{2, k}=\boldsymbol{C}_{21} \boldsymbol{x}_{1, k}+\boldsymbol{C}_{22} \boldsymbol{x}_{2, k}+\boldsymbol{\nu}_{2, k}
\end{aligned}
$$$$
\Delta^{\gamma_{2}} \boldsymbol{x}_{2, k}=\boldsymbol{A}_{21} \boldsymbol{x}_{1, k-1}+\boldsymbol{A}_{22} \boldsymbol{x}_{2, k-1}+\boldsymbol{B}_{2} \boldsymbol{u}_{k-1}+\boldsymbol{\omega}_{2, k-1}
$$

in that way that both subsystems are observable. $\boldsymbol{u}_{k-1}$ is a deterministic input in one or both subsystems, depending on $\boldsymbol{B} . \boldsymbol{x}_{1}$ is treated as an input for subsystem 2 . Based on this partition, two different local filters can be applied separately, with the second observer using the estimations of the first subsystem. As the local subsystems are smaller than the global model, the aim is to design simple and scalable observers for each subsystem. In general, the cascaded Kalman filter is applicable if the considered system can be represented by a directed acyclic graph, so that each node corresponds to an observable subsystem [13]. This is the same case for fractional order systems, because $\boldsymbol{\Gamma}_{i, j}$ with $i=1,2$ influences only local states in the particular subsystems due to its diagonal form. As a result, there is no need to communicate $\boldsymbol{\Gamma}_{i, j}$ between different subsystems.

The covariance matrices of the system and output noises are assumed to be block diagonal [9]. Otherwise, the value of the cross covariance between components that belong to different subsystems will be lost

$$
\boldsymbol{Q}=\left(\begin{array}{cc}
\boldsymbol{Q}_{1} & \mathbf{0} \\
\mathbf{0} & \boldsymbol{Q}_{2}
\end{array}\right) \quad \boldsymbol{R}=\left(\begin{array}{cc}
\boldsymbol{R}_{1} & \mathbf{0} \\
\mathbf{0} & \boldsymbol{R}_{2}
\end{array}\right)
$$

This assumption appears to be restrictive, but in practical applications, the value of the cross covariances are normally unknown and it is frequently assumed that the covariance matrices are diagonal [13]. Moreover, the output and system noises are assumed to be white, Gaussian, with zero mean and that different noise processes are uncorrelated.

\section{B. Cascaded State Estimation}

In order to estimate the local states of the first subsystem, which is given by (24) to (26), a linear Kalman filter is applied, based only on local measurements $\boldsymbol{y}_{1, k}$.

Theorem 1 (Kalman Filter for Subsystem 1). The Kalman gain, the state vector and state error covariance matrix for 
subsystem 1 can be calculated using the following formulas

$$
\begin{aligned}
\Delta^{\gamma_{1}} \hat{\boldsymbol{x}}_{1, k}^{-} & =\boldsymbol{A}_{11} \hat{\boldsymbol{x}}_{1, k-1}^{+}+\boldsymbol{B}_{1} \boldsymbol{u}_{k-1} \\
\hat{\boldsymbol{x}}_{1, k}^{-} & =\Delta^{\gamma_{1}} \hat{\boldsymbol{x}}_{1, k}^{-}-\sum_{j=1}^{z}(-1)^{j} \boldsymbol{\Gamma}_{1, j} \hat{\boldsymbol{x}}_{1, k-j}^{+} \\
\boldsymbol{P}_{11, k}^{-} & =\left(\boldsymbol{A}_{11}+\boldsymbol{\Gamma}_{1,1}\right) \boldsymbol{P}_{11, k-1}^{+}\left(\boldsymbol{A}_{11}+\boldsymbol{\Gamma}_{1,1}\right)^{T}+ \\
& +\boldsymbol{Q}_{1, k-1}+\sum_{j=2}^{z} \boldsymbol{\Gamma}_{1, j} \boldsymbol{P}_{11, k-j}^{+} \boldsymbol{\Gamma}_{1, j}^{T} \\
\boldsymbol{K}_{1, k} & =\boldsymbol{P}_{11, k}^{-} \boldsymbol{C}_{11}^{T}\left(\boldsymbol{C}_{11} \boldsymbol{P}_{11, k}^{-} \boldsymbol{C}_{11}^{T}+\boldsymbol{R}_{1}\right)^{-1} \\
\hat{\boldsymbol{x}}_{1, k}^{+} & =\hat{\boldsymbol{x}}_{1, k}^{-}+\boldsymbol{K}_{1, k}\left(\boldsymbol{y}_{1, k}-\boldsymbol{C}_{11} \hat{\boldsymbol{x}}_{1, k}^{-}\right) \\
\boldsymbol{P}_{11, k}^{+} & =\left(\boldsymbol{I}-\boldsymbol{K}_{1, k} \boldsymbol{C}_{11}\right) \boldsymbol{P}_{11, k}^{-}\left(\boldsymbol{I}-\boldsymbol{K}_{1, k} \boldsymbol{C}_{11}\right)^{T}+ \\
& +\boldsymbol{K}_{1, k} \boldsymbol{R}_{1} \boldsymbol{K}_{1, k}^{T} .
\end{aligned}
$$

Proof: Applying directly the formulas of the FKF from (12) to (17) to the equations of the first subsystem in (24) to (26) leads to the equations of Theorem 1.

In the second subsystem (27) to (29), $\boldsymbol{x}_{1, k}$ is an unknown stochastic variable and has therefore to be communicated from subsystem 1. In a distributed estimation algorithm, sometimes only the state estimation is communicated between different nodes or subsystems, and not the state error covariance matrix [13]. Therefore, similar to [13] two cases can be distinguished:

Case 1: Suppose that only the state estimation of $\boldsymbol{x}_{1, k}$ is transmitted from preceding subsystems and consider both $\boldsymbol{u}$ and $\hat{\boldsymbol{x}}_{1}$ as deterministic inputs in subsystem 2. In this case, the second subsystem will assume that the estimation provided by the first subsystem is correct, without randomness. However, the obtained state error covariance matrix in this case will not be the same as the true state error covariance matrix for the second subsystem. Following, the formulas of the Kalman filter for case 1 for the second subsystem is stated in Theorem 2.

Theorem 2 (Kalman Filter for Subsystem 2: Case 1). Let $\hat{\boldsymbol{x}}_{1}$ be seen as a deterministic input and apply a local Kalman filter to the second subsystem. Then the following equations are obtained

$$
\begin{aligned}
\Delta^{\gamma_{2}} \hat{\boldsymbol{x}}_{2, k}^{-} & =\boldsymbol{A}_{22} \hat{\boldsymbol{x}}_{2, k-1}^{+}+\boldsymbol{A}_{21} \hat{\boldsymbol{x}}_{1, k-1}^{+}+\boldsymbol{B}_{2} \boldsymbol{u}_{k-1} \\
\hat{\boldsymbol{x}}_{2, k}^{-} & =\Delta^{\gamma_{2}} \hat{\boldsymbol{x}}_{2, k}^{-}-\sum_{j=1}^{z}(-1)^{j} \boldsymbol{\Gamma}_{2, j} \hat{\boldsymbol{x}}_{2, k-j}^{+} \\
\boldsymbol{P}_{22, k}^{-} & =\left(\boldsymbol{A}_{22}+\boldsymbol{\Gamma}_{2,1}\right) \boldsymbol{P}_{22, k-1}^{+}\left(\boldsymbol{A}_{22}+\boldsymbol{\Gamma}_{2,1}\right)^{T}+ \\
& +\boldsymbol{Q}_{2, k-1}+\sum_{j=2}^{z} \boldsymbol{\Gamma}_{2, j} \boldsymbol{P}_{22, k-j}^{+} \boldsymbol{\Gamma}_{2, j}^{T} \\
\boldsymbol{K}_{2, k} & =\boldsymbol{P}_{22, k}^{-} \boldsymbol{C}_{22}^{T}\left(\boldsymbol{C}_{22} \boldsymbol{P}_{22, k}^{-} \boldsymbol{C}_{22}^{T}+\boldsymbol{R}_{2}\right)^{-1} \\
\hat{\boldsymbol{x}}_{2, k}^{+} & =\hat{\boldsymbol{x}}_{2, k}^{-}+\boldsymbol{K}_{2, k}\left(\boldsymbol{y}_{2, k}-\boldsymbol{C}_{21} \hat{\boldsymbol{x}}_{1, k}^{-}-\boldsymbol{C}_{22} \hat{\boldsymbol{x}}_{2, k}^{-}\right) \\
\boldsymbol{P}_{22, k}^{+} & =\left(\boldsymbol{I}-\boldsymbol{K}_{2, k} \boldsymbol{C}_{22}\right) \boldsymbol{P}_{22, k}^{-}\left(\boldsymbol{I}-\boldsymbol{K}_{2, k} \boldsymbol{C}_{22}\right)^{T}+ \\
& +\boldsymbol{K}_{2, k} \boldsymbol{R}_{2} \boldsymbol{K}_{2, k}^{T} .
\end{aligned}
$$

Proof: Applying directly the formulas of the FKF from (12) to (17) to the equations of the second subsystem in (27) to (29) leads to the equations of Theorem 2. The difference is that the formulas are indexed accordingly with their subsystem number and that $\hat{\boldsymbol{x}}_{1}$ is used as a deterministic input with a direct feedthrough on $\boldsymbol{y}_{2}$ in subsystem 2 .

Case 2: Assume that, in addition to the state estimation, the estimated covariance matrix $\boldsymbol{P}_{11}$ is communicated between subsequent modules. In this case, $\hat{\boldsymbol{x}}_{1}$ is considered as a stochastic variable in the second subsystem, with calculated covariance $\boldsymbol{P}_{11}$. Remark that only the estimated states and covariances will be sent from subsystem 1 to subsystem 2 . That means that all other information, such as the system matrices and measurements of other subsystems are not available in a particular subsystem and it is assumed that they are $\mathbf{0}$.

Theorem 3 (Kalman Filter for Subsystem 2: Case 2). Let $\hat{\boldsymbol{x}}_{1}$ be seen as a stochastic input with calculated covariance $\boldsymbol{P}_{11}$ and applying a local Kalman filter to the second subsystem. Then the following equations are obtained

$$
\begin{aligned}
\Delta^{\gamma_{2}} \hat{\boldsymbol{x}}_{2, k}^{-} & =\boldsymbol{A}_{22} \hat{\boldsymbol{x}}_{2, k-1}^{+}+\boldsymbol{A}_{21} \hat{\boldsymbol{x}}_{1, k-1}^{+}+\boldsymbol{B}_{2} \boldsymbol{u}_{k-1} \\
\hat{\boldsymbol{x}}_{2, k}^{-} & =\Delta^{\gamma_{2}} \hat{\boldsymbol{x}}_{2, k}^{-}-\sum_{j=1}^{z}(-1)^{j} \boldsymbol{\Gamma}_{2, j} \hat{\boldsymbol{x}}_{2, k-j}^{+} \\
\boldsymbol{P}_{22, k}^{-} & =\left(\boldsymbol{A}_{22}+\boldsymbol{\Gamma}_{2,1}\right) \boldsymbol{P}_{22, k-1}^{+}\left(\boldsymbol{A}_{22}+\boldsymbol{\Gamma}_{2,1}\right)^{T}+ \\
& +\sum_{j=2}^{z} \boldsymbol{\Gamma}_{2, j} \boldsymbol{P}_{22, k-j}^{+} \boldsymbol{\Gamma}_{2, j}^{T}+\boldsymbol{Q}_{2, k-1}+ \\
& +\boldsymbol{A}_{21} \boldsymbol{P}_{11, k-1}^{+} \boldsymbol{A}_{21}^{T}+ \\
& +\left(\left(\boldsymbol{A}_{22}+\boldsymbol{\Gamma}_{2,1}\right) \boldsymbol{P}_{21, k-1}^{+} \boldsymbol{A}_{21}^{T}\right) \\
& +\left(\left(\boldsymbol{A}_{22}+\boldsymbol{\Gamma}_{2,1}\right) \boldsymbol{P}_{21, k-1}^{+} \boldsymbol{A}_{21}^{T}\right)^{T} \\
\boldsymbol{K}_{2, k} & =\left(\boldsymbol{P}_{22, k}^{-} \boldsymbol{C}_{22}^{T}\right) \cdot \\
& \cdot\left(\boldsymbol{C}_{21} \boldsymbol{P}_{11, k}^{-} \boldsymbol{C}_{21}^{T}+\boldsymbol{C}_{22} \boldsymbol{P}_{22, k}^{-} \boldsymbol{C}_{22}^{T}+\boldsymbol{R}_{2}\right)^{-1} \\
\boldsymbol{P}_{22, k}^{+} & =\left(\boldsymbol{K}_{2, k} \boldsymbol{C}_{21}\right) \boldsymbol{P}_{11, k}^{-}\left(\boldsymbol{K}_{2, k} \boldsymbol{C}_{21}\right)^{T}+ \\
& +\left(\boldsymbol{I}-\boldsymbol{K}_{2, k} \boldsymbol{C}_{22}\right) \boldsymbol{P}_{22, k}^{-}\left(\boldsymbol{I}-\boldsymbol{K}_{2, k} \boldsymbol{C}_{22}\right)^{T}+ \\
& +\boldsymbol{K}_{2, k} \boldsymbol{R}_{2} \boldsymbol{K}_{2, k}^{T} \\
\boldsymbol{P}_{21, k}^{+} & =-\left(\boldsymbol{K}_{2, k} \boldsymbol{C}_{21}\right) \boldsymbol{P}_{11, k}^{-} \\
\hat{\boldsymbol{x}}_{2, k}^{+} & =\hat{\boldsymbol{x}}_{2, k}^{-}+\boldsymbol{K}_{2, k}\left(\boldsymbol{y}_{2, k}-\boldsymbol{C}_{21} \hat{\boldsymbol{x}}_{1, k}^{-}-\boldsymbol{C}_{22} \hat{\boldsymbol{x}}_{2, k}^{-}\right)
\end{aligned}
$$

This approach obtains the true state error covariance matrix for the second subsystem. 
Proof: First, we calculate $\boldsymbol{P}_{k}^{-}$using (14) as follows

$$
\begin{aligned}
& {\left[\begin{array}{ll}
\boldsymbol{P}_{11, k}^{-} & \boldsymbol{P}_{12, k}^{-} \\
\boldsymbol{P}_{21, k}^{-} & \boldsymbol{P}_{22, k}^{-}
\end{array}\right]} \\
& =\left[\begin{array}{cc}
\boldsymbol{A}_{11}+\boldsymbol{\Gamma}_{1,1} & \mathbf{0} \\
\boldsymbol{A}_{21} & \boldsymbol{A}_{22}+\boldsymbol{\Gamma}_{2,1}
\end{array}\right]\left[\begin{array}{cc}
\boldsymbol{P}_{11, k-1}^{+} & \boldsymbol{P}_{12, k-1}^{+} \\
\boldsymbol{P}_{21, k-1}^{+} & \boldsymbol{P}_{22, k-1}^{+}
\end{array}\right] \\
& \cdot\left[\begin{array}{cc}
\boldsymbol{A}_{11}^{T}+\boldsymbol{\Gamma}_{1,1} & \boldsymbol{A}_{21}^{T} \\
\mathbf{0} & \boldsymbol{A}_{22}^{T}+\boldsymbol{\Gamma}_{2,1}
\end{array}\right]+\left[\begin{array}{cc}
\boldsymbol{Q}_{1} & \mathbf{0} \\
\mathbf{0} & \boldsymbol{Q}_{2}
\end{array}\right] \\
& +\sum_{j=2}^{z}\left[\begin{array}{cc}
\boldsymbol{\Gamma}_{1, j} & \mathbf{0} \\
\mathbf{0} & \boldsymbol{\Gamma}_{2, j}
\end{array}\right]\left[\begin{array}{ll}
\boldsymbol{P}_{11, k-1}^{+} & \boldsymbol{P}_{12, k-1}^{+} \\
\boldsymbol{P}_{21, k-1}^{+} & \boldsymbol{P}_{22, k-1}^{+}
\end{array}\right]\left[\begin{array}{cc}
\boldsymbol{\Gamma}_{1, j} & \mathbf{0} \\
\mathbf{0} & \boldsymbol{\Gamma}_{2, j}
\end{array}\right] \\
& =\left[\begin{array}{ll}
\boldsymbol{Z}_{1,1} & \boldsymbol{Z}_{1,2} \\
\boldsymbol{Z}_{2,1} & \boldsymbol{Z}_{2,2}
\end{array}\right]+\left[\begin{array}{cc}
\boldsymbol{Q}_{1} & \mathbf{0} \\
\mathbf{0} & \boldsymbol{Q}_{2}
\end{array}\right] \\
& +\sum_{j=2}^{z}\left[\begin{array}{ccc}
\boldsymbol{\Gamma}_{1, j} \boldsymbol{P}_{11, k-1}^{+} \boldsymbol{\Gamma}_{1, j} & \boldsymbol{\Gamma}_{1, j} \boldsymbol{P}_{12, k-1}^{+} & \overbrace{\boldsymbol{\Gamma}_{2, j}}^{\text {unknown }} \\
\boldsymbol{\Gamma}_{2, j} \boldsymbol{P}_{21, k-1}^{+} \underbrace{\boldsymbol{\Gamma}_{1, j}}_{\text {unknown }} & \boldsymbol{\Gamma}_{2, j} \boldsymbol{P}_{22, k-1}^{+} \boldsymbol{\Gamma}_{2, j}
\end{array}\right]
\end{aligned}
$$

with

$$
\begin{aligned}
\boldsymbol{Z}_{1,1} & =\left(\boldsymbol{A}_{11}+\boldsymbol{\Gamma}_{1,1}\right) \boldsymbol{P}_{11, k-1}^{+}\left(\boldsymbol{A}_{11}^{T}+\boldsymbol{\Gamma}_{1,1}\right), \\
\boldsymbol{Z}_{1,2} & =\left(\boldsymbol{A}_{11}+\boldsymbol{\Gamma}_{1,1}\right) \boldsymbol{P}_{11, k-1}^{+} \overbrace{\boldsymbol{A}_{21}^{T}}^{\text {unknown }} \\
& +\left(\boldsymbol{A}_{11}+\boldsymbol{\Gamma}_{1,1}\right) \boldsymbol{P}_{12, k-1}^{+} \overbrace{\left(\boldsymbol{A}_{22}^{T}+\boldsymbol{\Gamma}_{2,1}\right)}^{\text {unknown }}, \\
\boldsymbol{Z}_{2,1} & =(\boldsymbol{A}_{21} \boldsymbol{P}_{11, k-1}^{+} \underbrace{\left(\boldsymbol{A}_{11}^{T}+\boldsymbol{\Gamma}_{1,1}\right)}_{\text {unknown }}) \\
& +\left(\boldsymbol{A}_{22}^{T}+\boldsymbol{\Gamma}_{2,1}\right) \boldsymbol{P}_{21, k-1}^{+} \underbrace{\left(\boldsymbol{A}_{11}^{T}+\boldsymbol{\Gamma}_{1,1}\right)}_{\text {unknown }}, \\
\boldsymbol{Z}_{2,2} & =\left(\boldsymbol{A}_{21} \boldsymbol{P}_{11, k-1}^{+}+\left(\boldsymbol{A}_{22}+\boldsymbol{\Gamma}_{2,1}\right) \boldsymbol{P}_{21, k-1}^{+}\right) \boldsymbol{A}_{21}^{T}+ \\
& +\boldsymbol{A}_{21} \boldsymbol{P}_{12, k-1}^{+}\left(\boldsymbol{A}_{22}^{T}+\boldsymbol{\Gamma}_{2,1}\right) \\
& +\left(\boldsymbol{A}_{22}+\boldsymbol{\Gamma}_{2,1}\right) \boldsymbol{P}_{22, k-1}^{+}\left(\boldsymbol{A}_{22}^{T}+\boldsymbol{\Gamma}_{2,1}\right) .
\end{aligned}
$$

Note that the parameters which are marked as "unknown" are set to $\mathbf{0}$ in the respective subsystem, because informations about the system parameters of other subsystems are not available as assumed before. Then it can be seen that $\boldsymbol{P}_{k}^{-}$is block diagonal. Also note that $\boldsymbol{\Gamma}_{i, j}=\boldsymbol{\Gamma}_{i, j}^{T} \forall i, j$, because they are diagonal matrices. Therefore $\boldsymbol{P}_{11}^{-}$and $\boldsymbol{P}_{22}^{-}$in (50) are identical to (33) and (45), respectively and $\boldsymbol{P}_{12}^{-}=\boldsymbol{P}_{21}^{-}=\mathbf{0}$. Next, the correction step of the state estimation can be proven straightforward using (16)

$\left[\begin{array}{l}\hat{\boldsymbol{x}}_{1, k}^{+} \\ \hat{\boldsymbol{x}}_{2, k}^{+}\end{array}\right]=\left[\begin{array}{l}\hat{\boldsymbol{x}}_{1, k}^{-} \\ \hat{\boldsymbol{x}}_{2, k}^{-}\end{array}\right]+\boldsymbol{K}_{k} \cdot\left(\left[\begin{array}{l}\hat{\boldsymbol{y}}_{1, k} \\ \hat{\boldsymbol{y}}_{2, k}\end{array}\right]-\left[\begin{array}{cc}\boldsymbol{C}_{11} & \mathbf{0} \\ \boldsymbol{C}_{21} & \boldsymbol{C}_{22}\end{array}\right] \cdot\left[\begin{array}{l}\hat{\boldsymbol{x}}_{1, k}^{-} \\ \hat{\boldsymbol{x}}_{2, k}^{-}\end{array}\right]\right)$

which yields (35) and (49).

Lemma 1. It also follows from (51) that $\boldsymbol{K}_{k}$ is blockdiagonal

$$
\boldsymbol{K}_{k}=\left[\begin{array}{cc}
\boldsymbol{K}_{1, k} & \mathbf{0} \\
\mathbf{0} & \boldsymbol{K}_{2, k}
\end{array}\right]
$$

since measurements of other subsystems are not considered.
The covariance matrix of the correction step is calculated using (18) and Lemma 1 to

$$
\begin{aligned}
& {\left[\begin{array}{ll}
\boldsymbol{P}_{11, k}^{+} & \boldsymbol{P}_{12, k}^{+} \\
\boldsymbol{P}_{21, k}^{+} & \boldsymbol{P}_{22, k}^{+}
\end{array}\right]} \\
& =\left(\boldsymbol{I}-\left[\begin{array}{cc}
\boldsymbol{K}_{1, k} & \mathbf{0} \\
\mathbf{0} & \boldsymbol{K}_{2, k}
\end{array}\right]\left[\begin{array}{ll}
\boldsymbol{C}_{11} & \mathbf{0} \\
\boldsymbol{C}_{21} & \boldsymbol{C}_{22}
\end{array}\right]\right) \cdot\left[\begin{array}{cc}
\boldsymbol{P}_{11, k}^{-} & \mathbf{0} \\
\mathbf{0} & \boldsymbol{P}_{22, k}^{-}
\end{array}\right] . \\
& \cdot\left(\boldsymbol{I}-\left[\begin{array}{cc}
\boldsymbol{K}_{1, k} & \mathbf{0} \\
\mathbf{0} & \boldsymbol{K}_{2, k}
\end{array}\right]\left[\begin{array}{ll}
\boldsymbol{C}_{11} & \mathbf{0} \\
\boldsymbol{C}_{21} & \boldsymbol{C}_{22}
\end{array}\right]\right)+ \\
& +\left[\begin{array}{cc}
\boldsymbol{K}_{1, k} & \mathbf{0} \\
\mathbf{0} & \boldsymbol{K}_{2, k}
\end{array}\right]\left[\begin{array}{cc}
\boldsymbol{R}_{1} & \mathbf{0} \\
\mathbf{0} & \boldsymbol{R}_{2}
\end{array}\right]\left[\begin{array}{cc}
\boldsymbol{K}_{1, k}^{T} & \mathbf{0} \\
\mathbf{0} & \boldsymbol{K}_{2, k}^{T}
\end{array}\right] \\
& =\left[\begin{array}{ccc}
\boldsymbol{W}_{1,1} & \boldsymbol{W}_{1,2} \\
\boldsymbol{W}_{2,1} & \boldsymbol{W}_{2,2}
\end{array}\right]+\left[\begin{array}{cc}
\boldsymbol{K}_{1, k} \boldsymbol{R}_{1} \boldsymbol{K}_{1, k}^{T} & \mathbf{0} \\
\mathbf{0} & \boldsymbol{K}_{2, k} \boldsymbol{R}_{2} \boldsymbol{K}_{2, k}^{T}
\end{array}\right]
\end{aligned}
$$

with

$$
\begin{aligned}
\boldsymbol{W}_{1,1} & =\left(\boldsymbol{I}-\boldsymbol{K}_{1, k} \boldsymbol{C}_{11}\right) \boldsymbol{P}_{11, k}^{-}\left(\boldsymbol{I}-\boldsymbol{K}_{1, k} \boldsymbol{C}_{11}\right)^{T}, \\
\boldsymbol{W}_{1,2} & =\left(\boldsymbol{I}-\boldsymbol{K}_{1, k} \boldsymbol{C}_{11}\right) \boldsymbol{P}_{11, k}^{-} \overbrace{\left(-\boldsymbol{K}_{2, k} \boldsymbol{C}_{21}\right)^{T}}^{\text {unknown }}, \\
\boldsymbol{W}_{2,1} & =\left(-\boldsymbol{K}_{2, k} \boldsymbol{C}_{21}\right) \boldsymbol{P}_{11, k}^{-}(\boldsymbol{I}-\underbrace{\boldsymbol{K}_{1, k} \boldsymbol{C}_{11}}_{\text {unknown }})^{T}, \\
\boldsymbol{W}_{2,2} & =\left(-\boldsymbol{K}_{2, k} \boldsymbol{C}_{21}\right) \boldsymbol{P}_{11, k}^{-}\left(-\boldsymbol{K}_{2, k} \boldsymbol{C}_{21}\right)^{T}+ \\
& +\left(\boldsymbol{I}-\boldsymbol{K}_{2, k} \boldsymbol{C}_{22}\right) \boldsymbol{P}_{22, k}^{-}\left(\boldsymbol{I}-\boldsymbol{K}_{2, k} \boldsymbol{C}_{22}\right)^{T}
\end{aligned}
$$

which results in (36), (47) and (48). It can be seen that a value for $\boldsymbol{P}_{21}^{+}$can be calculated in subsystem 2 , but as $\boldsymbol{K}$ is block diagonal it has no effect on the filter step, i.e. estimation of $\hat{\boldsymbol{x}}_{2}$, because just $\boldsymbol{P}_{22}^{+}$will be minimized. Nevertheless, it can still be used in the prediction step for the calculation of $\boldsymbol{P}_{22}^{-}$. Although $\boldsymbol{P}_{12}^{+}=\left(\boldsymbol{P}_{21}^{+}\right)^{T}$, it cannot be used by subsystem 1, because the missing system information $\boldsymbol{K}_{2, k}$ and $\boldsymbol{C}_{21}$ are assumed to be $\mathbf{0}$.

Finally, the Kalman gain can be computed by minimizing the trace of the error covariance for the second subsystem. Taking into account that matrices $\boldsymbol{P}$ and $\boldsymbol{R}$ are symmetric, equation (54) is obtained

$$
\begin{aligned}
\frac{\partial \boldsymbol{P}_{22, k}^{+}}{\partial \boldsymbol{K}_{2, k}} & =2 \boldsymbol{K}_{2, k} \boldsymbol{C}_{21} \boldsymbol{P}_{11, k}^{-} \boldsymbol{C}_{21}^{T}-2 \boldsymbol{P}_{22, k}^{-} \boldsymbol{C}_{22}^{T}+ \\
& +2 \boldsymbol{K}_{2, k} \boldsymbol{C}_{22} \boldsymbol{P}_{22, k}^{-} \boldsymbol{C}_{22}^{T}+2 \boldsymbol{K}_{2, k} \boldsymbol{R}_{2}= \\
& =2 \boldsymbol{K}_{2, k}\left(\boldsymbol{C}_{21} \boldsymbol{P}_{11, k}^{-} \boldsymbol{C}_{21}^{T}+\boldsymbol{C}_{22} \boldsymbol{P}_{22, k}^{-} \boldsymbol{C}_{22}^{T}+\boldsymbol{R}_{2}\right) \\
& -2 \boldsymbol{P}_{22, k}^{-} \boldsymbol{C}_{22}^{T} \stackrel{!}{=} \mathbf{0}
\end{aligned}
$$

Isolating $\boldsymbol{K}_{2, k}$, the previous equation results in (46).

Additionally, more consequences can be drawn from this section as follows in Corollary 1

Corollary 1. Comparing (53) with (17) and (50) with (14), it can be seen that the cascaded approach achieves the same error covariance matrix as the central Kalman filter, if and only if the subsystems are independent. That is, if $\boldsymbol{A}_{21}=\mathbf{0}, \boldsymbol{C}_{21}=\mathbf{0}$, and $\boldsymbol{R}_{12}=\mathbf{0}, \boldsymbol{Q}_{12}=\mathbf{0}$ as assumed in (30). Therefore, in general, the CFKF does not minimize the 
global state error covariance matrix, because it produces a systematic error and is hence suboptimal.

However, although the algorithm is not optimal, it usually performs sufficiently, because it can be assumed that the influence of the cross-covariances is negligible compared to the diagonal elements. Also note that in the cascaded Kalman filter, presented in [13], $\boldsymbol{P}_{21}^{+}$has been neglected unnecessarily.

Often, a distributed state estimation approach uses an explicit fusion step [10], [12]. In contrast, the CFKF uses an algorithm where the estimated states and covariances of preceding subsystems are directly used in the algorithm which makes it easier to implement.

\section{Generalization}

Considering more than two subsystems, one can extend the algorithm in the sections above as shown in Corollary 2.

Corollary 2 (Generalized Kalman Filter for Subsystem i: Case 2). Let there be more than 2 cascaded subsystems. Then, the algorithm of Theorem 1 and Theorem 3 can be extended to a generalized algorithm as follows

$$
\begin{aligned}
\Delta^{\gamma_{i}} \hat{\boldsymbol{x}}_{i, k}^{-}= & \boldsymbol{A}_{i i} \hat{\boldsymbol{x}}_{i, k-1}^{+}+\sum_{l=1}^{i-1} \boldsymbol{A}_{i l} \hat{\boldsymbol{x}}_{l, k-1}^{+}+\boldsymbol{B}_{i} \boldsymbol{u}_{k-1} \\
\hat{\boldsymbol{x}}_{i, k}^{-}= & \Delta^{\gamma_{i}} \hat{\boldsymbol{x}}_{i, k}^{-}-\sum_{j=1}^{z}(-1)^{j} \boldsymbol{\Gamma}_{i, j} \hat{\boldsymbol{x}}_{i, k-j}^{+} \\
\boldsymbol{P}_{i i, k}^{-}= & \left(\boldsymbol{A}_{i i}+\boldsymbol{\Gamma}_{i, 1}\right) \boldsymbol{P}_{i i, k-1}^{+}\left(\boldsymbol{A}_{i i}+\boldsymbol{\Gamma}_{i, 1}\right)^{T}+ \\
+ & \sum_{j=2}^{z} \boldsymbol{\Gamma}_{i, j} \boldsymbol{P}_{i i, k-j}^{+} \boldsymbol{\Gamma}_{i, j}^{T}+\boldsymbol{Q}_{i, k-1}+ \\
+ & \sum_{l=1}^{i-1} \boldsymbol{A}_{i l} \boldsymbol{P}_{l l, k-1}^{+} \boldsymbol{A}_{i l}^{T}+ \\
+ & \left(\sum_{l=1}^{i-1}\left(\boldsymbol{A}_{i i}+\boldsymbol{\Gamma}_{i, 1}\right) \boldsymbol{P}_{i l, k-1}^{+} \boldsymbol{A}_{i l}^{T}\right)^{T} \\
\hat{\boldsymbol{x}}_{i, k}^{+}= & \hat{\boldsymbol{x}}_{i, k}^{-}+\boldsymbol{K}_{i, k}\left(\boldsymbol{y}_{i, k}-\sum_{l=1}^{i-1} \boldsymbol{C}_{i l} \hat{\boldsymbol{x}}_{l, k}^{-}-\boldsymbol{C}_{i i} \hat{\boldsymbol{x}}_{i, k}^{-}\right) \\
+ & \left.\sum_{l=1}\left(\boldsymbol{A}_{i i}+\boldsymbol{\Gamma}_{i, 1}\right) \boldsymbol{P}_{i l, k-1}^{+} \boldsymbol{A}_{i l}^{T}\right)^{T} \\
\boldsymbol{P}_{i i, k}^{+}= & \sum_{l=1}^{i-1}\left(\boldsymbol{K}_{i, k} \boldsymbol{C}_{i l}\right) \boldsymbol{P}_{l l, k}^{-}\left(\boldsymbol{K}_{i, k} \boldsymbol{C}_{i l}\right)^{T}+ \\
+ & \left(\boldsymbol{P}_{i i, k}^{-} \boldsymbol{C}_{i i}^{T}\right) \\
\boldsymbol{K}_{i, k} & \left.\boldsymbol{K}_{i, k} \boldsymbol{C}_{i i}\right) \boldsymbol{P}_{i i, k}^{-}\left(\boldsymbol{I}_{i, k}^{T}-\boldsymbol{K}_{i, k} \boldsymbol{C}_{i i}\right)^{T}+ \\
& \left(\sum_{l=1}^{i-1}\left(\boldsymbol{C}_{i l} \boldsymbol{P}_{l l, k}^{-} \boldsymbol{C}_{i l}^{T}\right)+\boldsymbol{C}_{i i} \boldsymbol{P}_{i i, k}^{-} \boldsymbol{C}_{i i}^{T}+\boldsymbol{R}_{i}\right)
\end{aligned}
$$

where $l$ is the index for preceding subsystems with $l<i$.

The formulas of Corollary 2 can be derived, simply by applying the cascading algorithm to all subsystems successively.

\section{SIMULATION RESULTS}

In this section, we provide a comparison between the estimated states of the FKF, both cases of the CFKF and the state values from the simulation of a fractional order system. We consider a linear stochastic discrete fractional order state space system defined by

$\boldsymbol{A}=\left[\begin{array}{ccccc}-0.3 & 0.4 & 0 & 0 & 0 \\ -0.2 & -0.1 & 0 & 0 & 0 \\ 0 & 0.05 & -0.2 & -0.1 & 0 \\ 0 & 0.1 & 0.1 & -0.2 & 0 \\ 0 & -0.1 & 0 & -0.05 & 0.2\end{array}\right], \quad \boldsymbol{B}=\left[\begin{array}{c}0 \\ 0.1 \\ 0.1 \\ 0 \\ 0.1\end{array}\right]$

$\boldsymbol{C}=\left[\begin{array}{lllll}0 & 1 & 0 & 0 & 0 \\ 1 & 0 & 1 & 0 & 0 \\ 0 & 1 & 0 & 0 & 1\end{array}\right], \quad \gamma=\left[\begin{array}{l}0.7 \\ 1.2 \\ 0.8 \\ 0.5 \\ 0.2\end{array}\right]$

$\boldsymbol{R}=E\left[\boldsymbol{\nu}_{k} \boldsymbol{\nu}_{k}^{T}\right]=0.001 \boldsymbol{I}$

$\boldsymbol{Q}=E\left[\boldsymbol{\omega}_{k} \boldsymbol{\omega}_{k}^{T}\right]=0.001 \boldsymbol{I}$

$\boldsymbol{P}_{\left[-B_{L}, \ldots, 0\right]}=100 \boldsymbol{I}, \quad \boldsymbol{x}_{\left[-B_{L}, \ldots, 0\right]}=\left[\begin{array}{lllll}0 & 0 & 0 & 0 & 0\end{array}\right]^{T}$

with the sampling period $T=1 \mathrm{~s}$, the simulation time $T_{s}=300 \mathrm{~s}$ and the buffer length $B_{L}=300$. The covariance matrix $\boldsymbol{P}$ has large entries since the past states are unknown and assumed to zero. Following, our aim is to divide this system into subsystems to achieve an order reduction. One possibility is to divide the system into three cascaded subsystems $\boldsymbol{x}_{c}$ as follows

$$
\boldsymbol{x}_{c 1}=\left(\begin{array}{l}
x_{1} \\
x_{2}
\end{array}\right), \quad \boldsymbol{x}_{c 2}=\left(\begin{array}{l}
x_{3} \\
x_{4}
\end{array}\right), \quad \boldsymbol{x}_{c 3}=\left(x_{5}\right)
$$

with local measurements $y_{c}$

$$
y_{c 1}=y_{1}, \quad y_{c 2}=y_{2}, \quad y_{c 3}=y_{3} .
$$

The input and output signals of the described example are

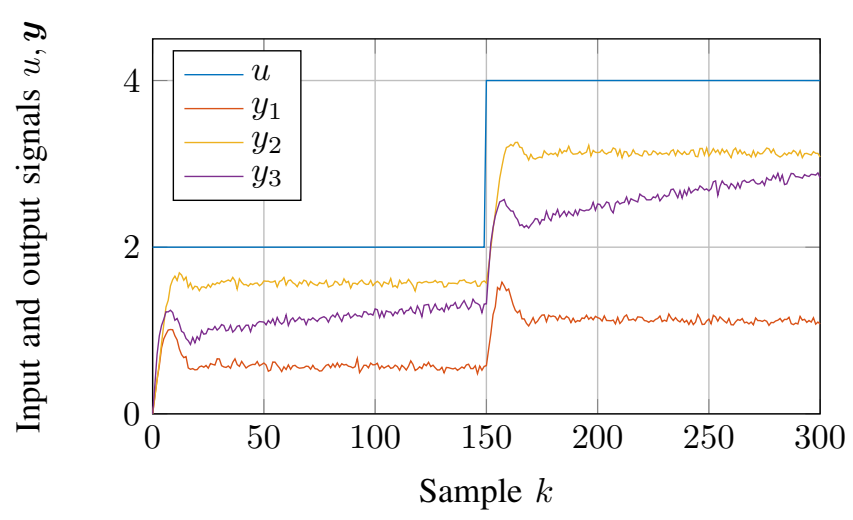

Fig. 1. Signal sequence of the input and outputs 


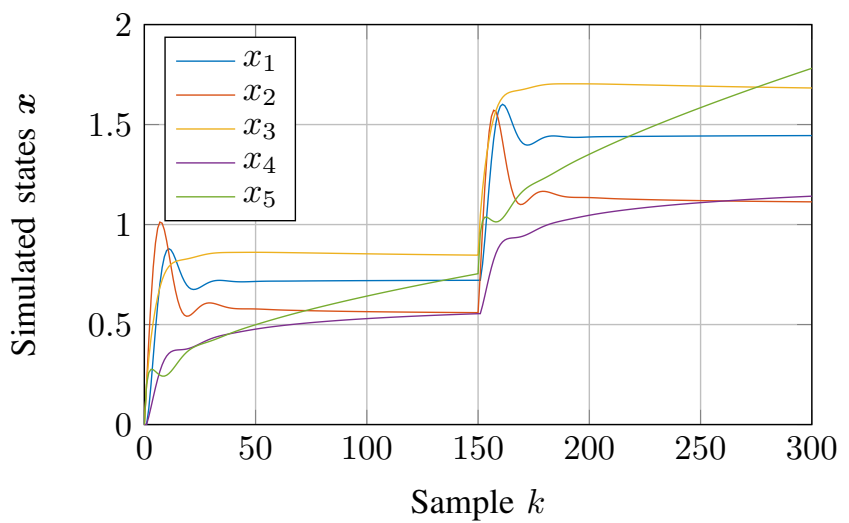

Fig. 2. Original state variables

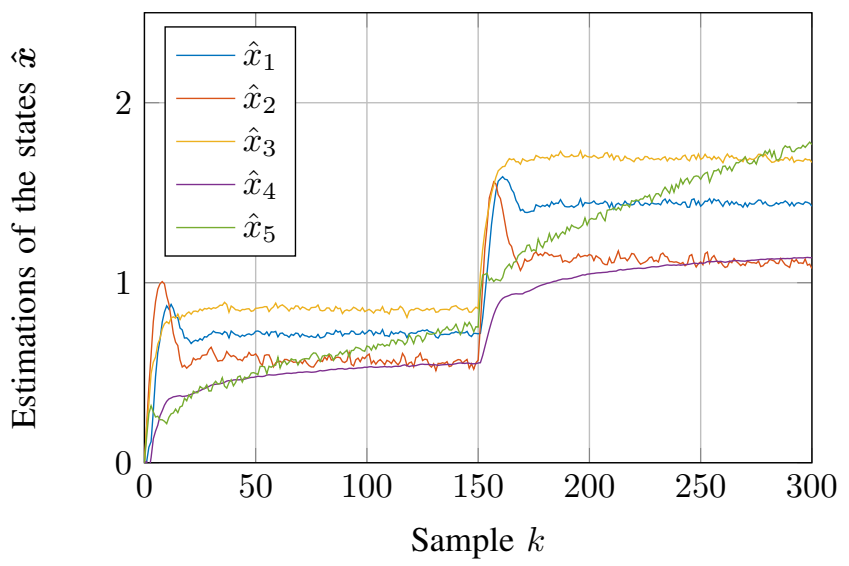

Fig. 3. Estimation of the states using the FKF

shown in Fig. 1 and the original state variables are given in Fig. 2. The signal to noise ratios of the output signals are calculated to $\mathrm{SNR}_{1}=28.7 \mathrm{~dB}, \mathrm{SNR}_{2}=38.5 \mathrm{~dB}$ and $\mathrm{SNR}_{3}=35.9 \mathrm{~dB}$ for $y_{1}, y_{2}$ and $y_{3}$. Since the main subject of this simulation is a comparison between the FKF and the CFKF, we use identical parameters. Comparing the results of the FKF in Fig. 3 with the original variables in Fig. 2, it can be seen that the estimation algorithm of the FKF works accurately. Comparing the estimation errors $e_{i}=\left(\hat{x}_{i}-x_{i}\right) / x_{i}$ of the FKF in Fig. 4 with case 1 of the CFKF given in Fig. 5 and with case 2 of the CFKF given in Fig. 6, it can be seen that all three algorithms lead to similar results. The main difference is that the CFKF converges some steps slower to a given error level due to missing information. In most applications, this effect does not yield problems. Further, the Root Mean Square Error (RMSE) of all three algorithms, averaged for 100 simulation runs is presented in Table I. It can be seen that the RMSE of CFKF case 2 is very similar to the FKF. As expected, the RMSE of $x_{3}, x_{4}$ and $x_{5}$ of CFKF case 1 is worse due to the missing covariance information of preceding subsystems. In this example, subsystem 2 needs information of subsystem 1 , and subsystem 3 needs data from both subsystems 1 and 2 . Note that in other examples an all-

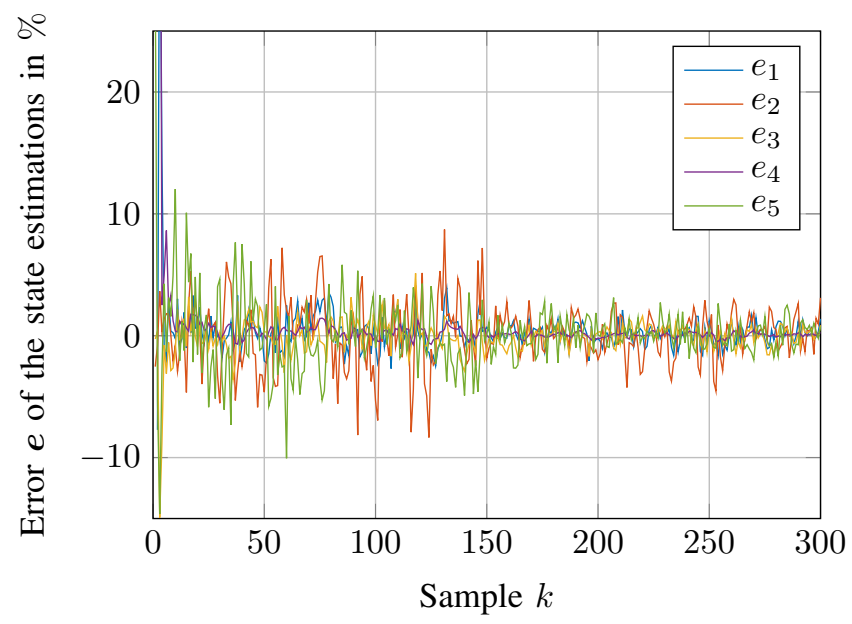

Fig. 4. Estimation errors of the states in percent using the FKF

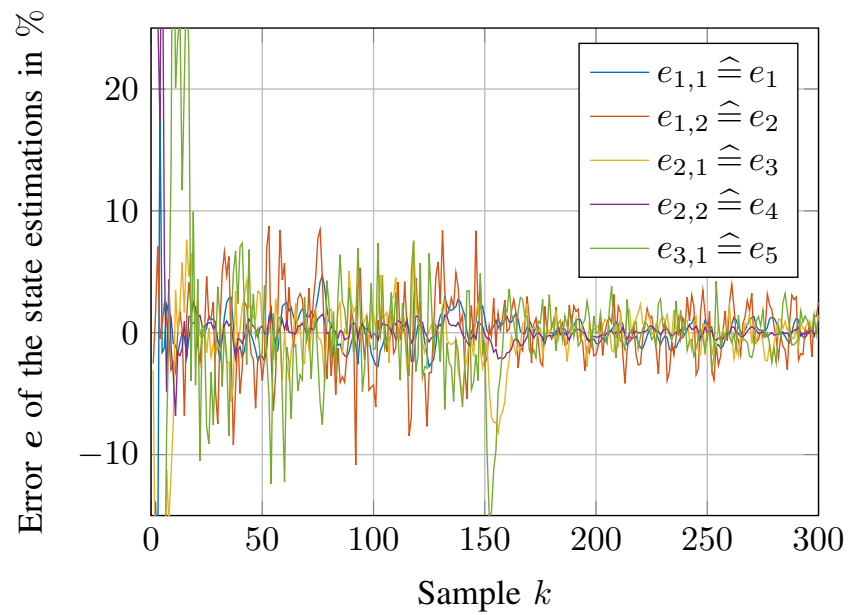

Fig. 5. Estimation errors of the states in percent using the CFKF case 1

to-all connection between all subsystems is not necessarily needed! In this example that would be the case for $A_{52}=$ $C_{32}=0$.

In a second example, we want to show the performance of the filters in case of a wrong initialization of the states. For this reason we initialize the filters with

$$
\boldsymbol{P}_{\left[-B_{L}, \ldots, 0\right]}=100 \boldsymbol{I}, \quad \hat{\boldsymbol{x}}_{\left[-B_{L}, \ldots, 0\right]}=\left[\begin{array}{lllll}
4 & 4 & 4 & 4 & 4
\end{array}\right]^{T}
$$

while the simulation still starts with $\boldsymbol{x}_{\left[-B_{L}, \ldots, 0\right]}=\mathbf{0}$. The averaged RMSE for 100 simulation runs is given in Table II. The estimated states of the CFKF case 2 are shown in Fig. 7. It can be seen that the filters still converge, but they need more time to reach a given error level due to the memory effect, as expected.

\section{CONCLUSION}

This article presents a distributed Kalman filter for cascaded fractional order systems. If the system matrices can be transformed into block lower triangular matrices, it is often convenient to divide the model into cascaded subsystems. 


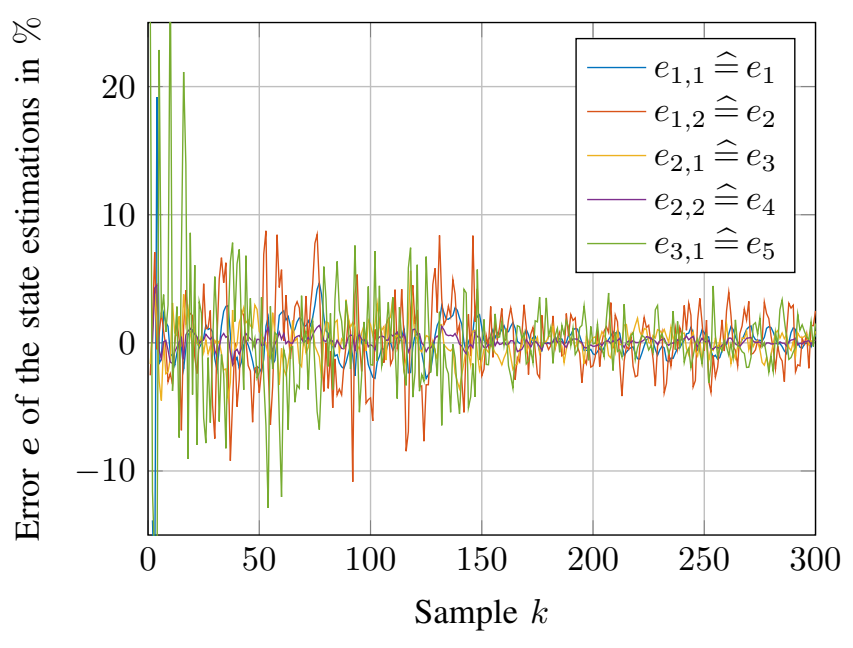

Fig. 6. Estimation errors of the states in percent using the CFKF case 2

TABLE I

RMSE OF THE FILTERS, AVERAGED FOR 100 RUNS FOR EXAMPLE 1

\begin{tabular}{c||c|c|c|c|c} 
& $x_{1}$ & $x_{2}$ & $x_{3}$ & $x_{4}$ & $x_{5}$ \\
\hline \hline RMSE FKF & 0.0158 & 0.0186 & 0.0157 & 0.0104 & 0.0174 \\
\hline RMSE CFKF1 & 0.0132 & 0.0223 & 0.0313 & 0.0073 & 0.0389 \\
\hline RMSE CFKF2 & 0.0132 & 0.0223 & 0.0147 & 0.0030 & 0.0245 \\
\hline
\end{tabular}

TABLE II

RMSE OF THE FILTERS, AVERAGED FOR 100 RUNS FOR EXAMPLE 2

\begin{tabular}{c||c|c|c|c|c} 
& $x_{1}$ & $x_{2}$ & $x_{3}$ & $x_{4}$ & $x_{5}$ \\
\hline \hline RMSE FKF & 0.2865 & 0.2366 & 0.2890 & 0.7851 & 0.2499 \\
\hline RMSE CFKF1 & 0.3813 & 0.2327 & 0.4401 & 0.7086 & 0.3270 \\
\hline RMSE CFKF2 & 0.3813 & 0.2327 & 0.3414 & 0.7085 & 0.3148 \\
\hline
\end{tabular}

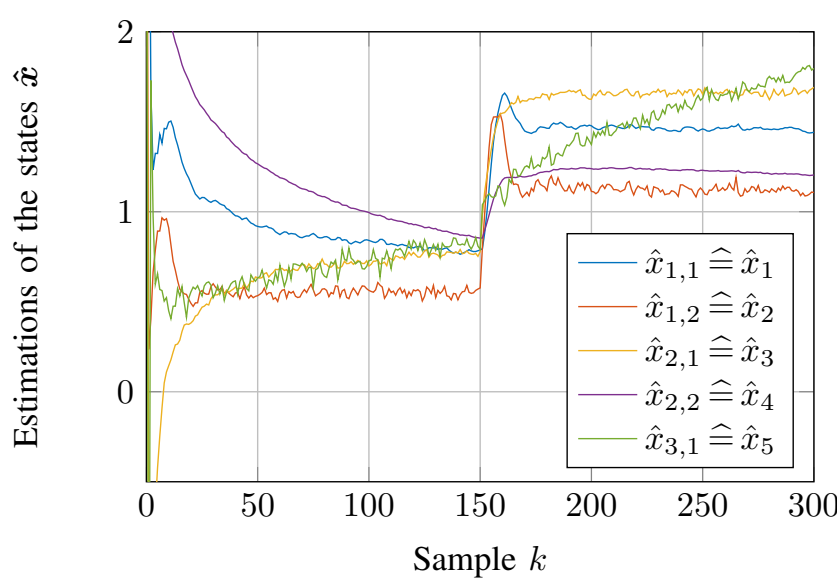

Fig. 7. Estimation of the states using the CFKF case 2 for example 2

Such a distribution leads to small and scalable nodes which is advantageous, especially in case of computationally intensive models like fractional systems. Two cases for the CFKF are distinguished, one where the estimated states are commu- nicated as deterministic variables and one where they are communicated as stochastic variables. A simulation example has been given which compares both cases together with the FKF. It could be seen that all algorithms lead to comparable results. In a second example, it could be seen that a wrong initialization could also be managed by the filters.

In the future, we want to implement this procedure for the distributed state estimation of large battery systems where the cells have been modelled using fractional impedances.

\section{REFERENCES}

[1] J. Sabatier, M. Merveillaut, J. M. Francisco, F. Guillemard, and D. Porcelatto, "Fractional models for lithium-ion batteries." in $E u$ ropean Control Conference (ECC), Zürich, Switzerland, 2013.

[2] J. Illig, T. Chrobak, M. Ender, J. P. Schmidt, D. Klotz, and E. IversTiffee, "Studies on lifepo4 as cathode material in li-ion batteries," ECS Transactions, vol. 28, no. 30, pp. 3-17, october 2010.

[3] M. Eckert, M. Kupper, and S. Hohmann, "Functional fractional calculus for system identification of battery cells." Automatisierungstechnik, vol. 62, no. 62, pp. 272-281, March 2014.

[4] M. Eckert, L. Kölsch, and S. Hohmann, "Fractional algebraic identification of the distribution of relaxation times of battery cells," in 54th IEEE Conference on Decision and Control (CDC), Osaka, Japan, 2015.

[5] A. Dabiri, M. Nazari, and E. Butcher, "The spectral parameter estimation method for parameter identification of linear fractional order systems," in American Control Conference (ACC), 2016.

[6] C. A. Monje, Y. Chen, B. M. Vinagre, D. Xue, and V. Feliu, Fractional-order Systems and Controls. Springer, 2010.

[7] D. Sierociuk and A. Dzielinski, "Fractional kalman filter algorithm for the states, parameters and order of fractional system estimation." International Journal of Applied Mathematics and Computer Science, vol. 16, no. 1, pp. 129 - 140, 2006.

[8] I. N'Doye, H. Voos, T.-M. Laleg-Kirati, and M. Darouach, " $H$ infinity adaptive observer design and parameter identification for a class of nonlinear fractional-order systems," in 53rd IEEE Conference on Decision and Control (CDC), Los Angeles, CA, USA, 2014

[9] P. Hilgers, "Verteilte zustandsschätzung nichtlinearer systeme." Universitatsverlag Ilmenau, 2012.

[10] P. Hilgers and C. Ament, "Distributed and decentralized estimation of non-linear systems." in Proceedings of the IEEE International Conference on Control Applications, Yokohama, Japan, 2010, pp. 328 -333 .

[11] A. G. O. Mutambara and H. F. Durrant-Whyte, "Modular scalable robot control," in Multisensor Fusion and Integration for Intelligent Systems, 1994. IEEE International Conference on MFI '94, Oct 1994, pp. 121-127.

[12] M. Kupper, I. Sesar Gil, and S. Hohmann, "Distributed and decentralized state estimation of fractional order systems," in American Control Conference, ACC 2016, Boston, MA, USA, 2016.

[13] Z. Lendek, R. Babuška, and B. De Schutter, "Distributed kalman filtering for cascaded systems," Engineering applications of artificial intelligence, vol. 21, pp. $457-569,2008$.

[14] S. Pequito, P. Bogdan, and G. J. Pappas, "Minimum number of probes for brain dynamics observability," in 54th IEEE Conference on Decision and Control (CDC), Osaka, Japan, 2015.

[15] I. Podlubny, Fractional differential equations, ser. Mathematics in Science and Engineering. Academic Press, 1999.

[16] S. Guermah, S. Djennoune, and M. Bettayeb, "Discrete-time fractional-order systems: Modeling and stability issues," in Advances in Discrete Time Systems, M. Mahmoud, Ed. InTech, 2012.

[17] C. F. Lorenzo and T. T. Hartley, Initialized fractional calculus. National Aeronautics and Space Administration, Glenn Research Center, 2000.

[18] J. Sabatier, C. Farges, and J.-C. Trigeassou, "Fractional systems state space description: some wrong ideas and proposed solutions," Journal of Vibration and Control, vol. 20, no. 7, pp. 1076-1084, 2014.

[19] D. Sierociuk, I. Tejado, and B. M. Vinagre, "Improved fractional kalman filter and its application to estimation over lossy networks." Signal Processing, an international journal., vol. 91, no. 3, pp. $542-$ 552, March 2011. 